\title{
Análise dos determinantes do nível de felicidade subjetiva: uma abordagem local
}

\author{
Analysis of the determinants of the level of subjective happiness: a local approach
} Analyse des déterminants du niveau de bonheur subjectif: une approche locale Análisis de los determinantes del nivel de felicidad subjetiva: un enfoque local

\author{
Leonardo Recena Aydos* \\ Leonardo Francisco Figueiredo Neto* \\ Wladimir Machado Teixeira*
}

Recebido em 17/04/2016; revisado e aprovado em 06/10/2016; aceito em 06/10/2016

DOI: http:/ / dx.doi.org/10.20435/1984-042X-2017-v.18-n.1(11)

\begin{abstract}
Resumo: A Economia da Felicidade é uma área interdisciplinar envolvendo psicologia, sociologia, economia, entre outras. O presente estudo buscou analisar determinantes econômicos e sociais que explicam os níveis de felicidade da população estudada. Um questionário no estilo survey foi aplicado a 600 indivíduos do município de Campo Grande, MS. Um modelo econométrico multinomial não linear apontou que a escolaridade, a idade, o estado civil e o Estado de origem são variáveis que influenciam nos níveis de felicidade.
\end{abstract}

Palavras-chave: economia da felicidade; Probit ordenado; bem-estar subjetivo.

Abstract: The Economics of Happiness is an interdisciplinary area involving Psychology, sociology, economics, among others. This study investigates economic and social determinants that explain the happiness levels of a population. A questionnaire was administered to 600 individuals in Campo Grande Municipality, Mato Grosso do Sul State, Brazil. A non linear multinomial model showed that education, age, marital status and country of origin are variables that influence the levels of happiness.

Key words: economics of happiness; ordered Probit; subjective well-being.

Résumé: L'économie du bonheur c'est un domaine interdisciplinaire qui implique la psychologie, la sociologie, l'économie entre autres. Cette étude examine les déterminants économiques et sociaux qui expliquent les niveaux de bonheur d'une certaine population, qui a été l'objet de l'étude. Un questionnaire dans un style d'enquête a été appliqué à 600 personnes à Campo Grande, Ville de l'Etat du Mato Grosso de Sul, Brésil. Un modèle économétrique multinomial non linéaire a montré que l'éducation, l'âge, l'état civil et le pays d'origine sont des variables qui influencent les niveaux de bonheur.

Mots-clés: économie de bonheur; Probit ordonné; bien-être subjectif.

Resumen: La economía de lafelicidad es un área interdisciplinaria que involucra lapsicología, lasociología, laeconomía, entre otros. Este estudio investiga los determinantes económicos y sociales que explicanlos niveles de felicidad de lapoblaciónestudiada. Uncuestionarioenel estilo de levantamiento se aplicó a 600 personas en Campo Grande, MS, Brasil. Un modelo econométrico multinomial no lineal mostró que laeducación, laedad, el estado civil y el país de origensonlasvariables que influyenenlos niveles de felicidad.

Palabras clave: economía de la felicidade; Probit ordenado; bienestar subjetivo.

\section{INTRODUÇÃO}

Ao longo da história, os filósofos têm considerado a busca pela felicidade a principal motivadora das ações humanas (DIENER, 1984, p. 542). Porém a mensuração da felicidade de um indivíduo é complexa, pois, como afirmam Corbi e Menezes-Filho (2006), o bem-estar ${ }^{1}$ pode ser analisado sob duas abordagens - a objetiva e a subjetiva. Pela abordagem

\footnotetext{
${ }^{1}$ Este trabalho considera sinônimos os conceitos de bem-estar subjetivo e felicidade subjetiva.
}

* Universidade Federal de Mato Grosso do Sul (UFMS), Campo Grande, Mato Grosso do Sul, Brasil. 
objetiva, o bem-estar/felicidade pode ser aferido por indicadores sociais, de saúde, criminalidade, entre outros. Já pela abordagem subjetiva, que "[...] consiste na experiência interna de cada indivíduo, isto é, tudo aquilo que passa em sua mente de forma espontânea, que ele sente e pensa sobre a vida que tem levado" (CORBI; MENEZES-FILHO, 2006, p. 520), não temos indicadores definidos. Dessa forma, "baseados na vasta experiência dos psicólogos, economistas perceberam que uma boa forma de acessar o bem-estar de uma pessoa é, simplesmente, perguntando a ela" (NETTO, 2014, p. 47). Espera-se que quanto maior o bem-estar objetivo maior seja o subjetivo, porém, em alguns casos, mesmo com condições adequadas de saúde, moradia e outros indicadores objetivos, uma pessoa pode não se definir como feliz.

O presente trabalho contribui para essa discussão, trazendo reflexões alicerçadas numa base de dados primária, com significância estatística, fruto de questionários no estilo survey, e utiliza como método para análise dos dados um modelo de Probit Ordenado. Assim, procurou-se responder ao seguinte problema: "qual a influência de determinantes socioeconômicos nos níveis de felicidade dos moradores de Campo Grande, MS?". E buscou-se indicar determinantes socioeconômicos que influenciam o nível de felicidade dos indivíduos para auxiliar políticas sociais, possibilitando maior retorno em relação aos investimentos.

\section{ESTUDOS SOBRE “A FELICIDADE"}

Os estudos sobre a Economia da Felicidade utilizam alguns conceitos e ferramentas da área de ciências econômicas, sociologia, ciência política e da psicologia para analisar os fatores relacionados ao bem-estar das pessoas. Segundo Nery (2014), "Na maioria desses estudos, dados provenientes de surveys amplos e representativos são analisados com mé- todos estatísticos, de modo a observar a correlação entre os níveis de felicidade e fatores (econômicos ou não) da vida dos indivíduos".

Corbi e Menezes-Filho (2006), utilizando dados do World Values Survey (WVS), fizeram um estudo que verificou em cinco países - com uma análise mais aprofundada no Brasil - como algumas variáveis econômicas e demográficas influenciam no bem-estar dos indivíduos. Assim, estimaram o nível de felicidade dos indivíduos estudados, utilizando um modelo multinominal, em que "sua variável dependente assume valores que estabelecem certo ordenamento dos dados, não de forma linear, mas de forma a ranquear os possíveis resultados" (CORBI; MENEZES-FILHO, 2006).

Lima (2007), através da mesma base de dados (WVS) e modelo, fez uma pesquisa que verificou a influência de determinantes empíricos na felicidade dos brasileiros, sendo que "os determinantes considerados foram estado civil, idade, escolaridade, sexo, região, religião, etnia, renda, posição relativa da renda, desemprego, probabilidade de desemprego para indivíduos empregados e probabilidade de emprego para indivíduos desempregados" (LIMA, 2007, p. 8). Helliwell (2003), utilizando dados do WSV dos anos de 1980-1982, 1990-1991 e 1995-1997, estudou o bem-estar subjetivo de indivíduos de 49 países diferentes.

Borrero et al. (2013, p.2) analisaram os moderadores situacionais e culturais da relação entre riqueza e felicidade de 197 países, utilizando como método a análises de variância para verificar como "a pobreza - tal como outras situações adversas - tem efeito negativo sobre a felicidade e que esse efeito é atenuado por uma orientação coletivista".

Scalco, Araújo e Bastos (2011) fizeram um estudo no município de Pelotas, RS, em que, através da coleta de dados por meio de questionários, analisaram a influência de algumas variáveis 
sociodemográficas sobre a autopercepção de felicidade dos indivíduos estudados.

Rodrigues e Silva (2010) fizeram uma pesquisa com o objetivo de verificar a influência de algumas variáveis sobre o bem-estar subjetivo de trabalhadores do comércio no município de Ribeirão Preto e região.

Diener (1984) e Ferraz, Tavares e Zilberman (2007) analisaram e procederam a uma revisão de literatura ligada ao tema da felicidade, bem-estar e satisfação de vida. Caldas (2010) verificou a relação entre felicidade e consumo.

Esses estudos indicam relações entre a percepção de felicidade subjetiva com variáveis sociodemográficas e econômicas e serviram de balizamento para nossa pesquisa, tanto na constituição do instrumento de coleta de dados como na discussão dos resultados encontrados.

\section{METODOLOGIA}

\subsection{Município estudado e amostra}

A amostra para representar a população de Campo Grande,MS, com 95\% de confiança e $4 \%$ de erro, foi calculada pela equação 15:

$$
n=\frac{N \cdot Z^{2} \cdot p \cdot(q)}{Z^{2} \cdot p \cdot(1-p)+e^{2} \cdot(N-1)}
$$

Em que $n$ é a amostra; $N$, a população; $Z$, o valor crítico que corresponde ao grau de confiança desejado; $\boldsymbol{p}, \boldsymbol{a}$ proporção populacional de indivíduos que pertencem à categoria estudada; $\boldsymbol{q}$, a proporção populacional de indivíduos que não pertencem à categoria estudada; e $\boldsymbol{e}$ é $\boldsymbol{o}$ erro da amostra.

Assim, foram adotados os valores: $n$ (Amostra necessária); $N$ (786.797 moradores); $Z$ (1,96, valor crítico de $Z a / 2$, para um grau de confiança de $95 \%) ; p$ $(0,5) ; \boldsymbol{q}(0,5)$ e $\boldsymbol{e}(0,04,4 \%$ de erro para mais ou para menos). Após o cálculo, foi determinado que a amostra mínima para obter um erro amostral de $4 \%$ a $95 \%$ de confiança deveria ser de 600 moradores.

Os questionários foram aplicados durante os meses de junho a agosto de 2014, no município de Campo Grande, Estado de Mato Grosso do Sul. Segundo o IBGE, o município tinha em 2010, 786.797 habitantes, o que representa $32,1 \%$ da população do Estado, estando 98,66\% dos habitantes localizados na zona urbana do município (SAUER; CAMPELO; CAPILLÉ, 2012). Para obter uma amostra que representasse com maior fidelidade a realidade dos moradores do município estudado, os 600 questionários foram aplicados em 10 bairros de Campo Grande, definidos de acordo com o índice de exclusão social do município.

O Índice de Exclusão Social de Campo Grande é composto pelas variáveis Indicador de Pobreza, Indicador de Desigualdade, Razão de Dependência Demográfica, Taxa de Alfabetização, Indicador de Escolaridade, Indicador de direitos suprimidos para crianças e jovens e Participação de Jovens. Esse índice ocorre de forma radial, como acontece na maioria das cidades de médio e pequeno porte do Brasil: o centro, com os menores índices, caminhando para a periferia, com os maiores índices (SAUER; CAMPELO; CAPILLÉ, 2012).

Assim, foram estipulados 120 questionários para cada dois bairros de cada um dos cinco níveis de exclusão social de Campo Grande. Esses questionários foram aplicados em cada um dos seguintes bairros: Jardim dos Estados e Bela Vista, que têm Índice de Exclusão Social entre 0,05 e 0,21; Vilas Boas e TV Morena, com Índice de Exclusão Social entre 0,21 e 0,45; Amambai e Carlota, com Índice de Exclusão Social entre 0,45 e ,061; Piratininga e Aero Rancho, com Índice de Exclusão Social entre 0,61 e 0,74; e Centro Oeste e Los Angeles, com Índice de Exclusão Social entre 0,74 e 0,96. 


\section{$3.2 \mathrm{O}$ modelo}

Neste estudo, a felicidade dos indivíduos foi aferida em quatro escalas: infeliz, não muito feliz, feliz e muito feliz. Ou seja, a variável dependente é discreta e ordenada, tendo sido utilizado um modelo de Probit ordenado para analisar os dados. Guo e Hu (2011) defendem que, quando as respostas para a felicidade dos indivíduos são ordinais e não cardinais, deve-se utilizar um modelo de Probit/ Logit ordenado para melhor estimá-las. Gujarati e Porter (2011) apontam que, quando se trata de escalas ordinais, em que existe uma hierarquia entre as categorias, devem ser utilizados modelos Logit/ Probit ordenados na análise estatística.

Nesta pesquisa, a variável a ser explicada foi dividida em quatro níveis: 1 = Infeliz; 2 = Não muito feliz; 3 = Feliz; e $4=$ Muito Feliz. Portanto existe uma hierarquia bem definida entre as variáveis, porém não é possível afirmar que 3 seja o triplo de 1, por isso é adequada a utilização do modelo Probit ordenado, já que ele não classifica necessariamente um valor 2 como sendo simplesmente o dobro do valor 1 .

Greene (2002) aponta que o modelo Probit Ordenado é construído em torno da regressão latente abaixo:

$y^{*}=x^{\prime} \beta+\mathbf{s}$

Sendo que $\mathrm{y}^{*}$ não é observado, assim, o observado será:

$y=0$ se $y^{*} \leq \mathbf{0}$

$y=1$ se $0<y^{*} \leq \mu_{1}$

$y=2$ se $\mu_{1}<y^{*} \leq \mu_{\mathbf{2}}$

$y=3$ se $\mu_{\mathbf{2}}<y^{*} \leq \mu_{\mathbf{3}}$

$y=4$ se $\mu_{3} \leq y^{*}$

Se assumirmos que $\mathbf{s}$ tem distribuição normal em todas as observações e normalizarmos a média e a variância para 1 e 0 , então teremos:

$$
\begin{aligned}
& \operatorname{Prob}(y=\mathbf{0} \mid x)=\boldsymbol{\Phi}\left(-x^{\prime} \beta\right) \text {, } \\
& \operatorname{Prob}(y=1 \mid x)=\varnothing\left(\mu_{1}=x^{\prime} \beta\right)-\varnothing\left(-x^{\prime} \beta\right) \\
& \operatorname{Prob}(y=2 \mid x)=\varnothing\left(\mu_{2}=x^{\prime} \beta\right)-\sigma\left(\mu_{1}-x^{\prime} \beta\right) \\
& \operatorname{Prob}(y=3 \mid x)=\varnothing\left(\mu_{3}=x^{\prime} \beta\right)-\varnothing\left(\mu_{2}-x^{\prime} \beta\right) \\
& \operatorname{Prob}(y=\mathbf{4} \mid x)=\boldsymbol{\sigma}\left(\mu_{4}=x^{\prime} \beta\right)-\varnothing\left(\mu_{3}-x^{\prime} \beta\right)
\end{aligned}
$$

Por fim, a probabilidade dos efeitos marginais será dada por:

$\frac{\partial \operatorname{Prob}(y=0 \mid x)}{\partial x}=-\boldsymbol{\Phi}\left(x^{\prime} \beta\right) \beta$,

$\frac{\partial \operatorname{Prob}(y=1 \mid x)}{\partial x}=\left[\varnothing\left(-x^{\prime} \beta\right)-\varnothing\left(\mu-x^{\prime} \beta\right)\right] \beta$,

$\frac{\partial \operatorname{Prob}(y=2 \mid x)}{\partial x}=\boldsymbol{\sigma}\left(\mu-x^{\prime} \beta\right) \beta$.

Primeiramente, os dados coletados nos questionários foram tabulados no software Excel 2013, em seguida, o software estatístico Stata SE, versão 12, foi utilizado para rodar o modelo. 


\subsection{Instrumento de coleta de dados}

Os dados foram coletados por meio de questionários no estilo survey, método mundialmente utilizado por possibilitar que o pesquisador consiga coletar dados de distintas variáveis importantes para a pesquisa e, posteriormente, transformá-los em forma numérica para proceder às análises estatísticas (HYMAN, 1967), analisando uma amostra predefinida pelo pesquisador, diferentemente das pesquisas do tipo censo, que são focadas em toda a população (BABBIE, 1999).

O questionário aplicado procurou obter informações sobre as seguintes características dos entrevistados: i) gênero; ii) idade; iii) escolaridade; iv) estado Civil; v) unidade federativa (estado) de origem; vi) situação no mercado de trabalho; vii) renda; e viii) nível de felicidade subjetiva.

A entrevista foi feita nos meses de janeiro a março de 2014, em diferentes períodos do dia, de acordo a disponibilidade do entrevistado, de forma pessoal e individualizada.
$\mathrm{O}$ método mais comum para verificação do bem-estar subjetivo de um indivíduo é através de escalas de autoavaliação. De fato, o que se verifica é que o método a ser utilizado para aferir a felicidade em um indivíduo depende do propósito que o autor objetiva ao fazer seu estudo (DIENER, 1984).

Neste trabalho, foi utilizada a "escala com item único", com a seguinte pergunta: considerando todas as coisas, $\mathrm{o}$ quão feliz você está nos dias atuais? Para tanto, utilizou-se um intervalo, que compreende as seguintes possíveis respostas: "infeliz"; "não muito feliz"; "feliz"; e "muito feliz".

\section{RESULTADOS E DISCUSSÃO}

Nesta seção, são discutidos os resultados da pesquisa assim como sua comparação com as conclusões de outros trabalhos. A Tabela 1 mostra os resultados do modelo de Probit que teve como variável dependente o nível de felicidade dos entrevistados.

Tabela 1 - Resultados do Modelo - Campo Grande, 2014

\begin{tabular}{lccccc}
\hline \multicolumn{1}{c}{ Variáveis } & Coeficientes & $\begin{array}{c}\text { Des. Padrão } \\
\text { Rob. }\end{array}$ & $\mathbf{P}>\mathbf{z}$ & $\begin{array}{c}\text { [95\%. Intervalo } \\
\text { Conf.] }\end{array}$ & \\
\hline Quartil Renda 2 & .1044908 & .1505398 & 0.488 & -.1905617 & .3995433 \\
Quartil Renda 3 & -.0040577 & .1477928 & 0.978 & -.2937263 & .2856109 \\
Quartil Renda 4 & .1230581 & .181581 & 0.498 & -.2328342 & .4789504 \\
Empregado & .2605184 & .1761251 & 0.139 & -.0846805 & .6057173 \\
Aposentado & -.086827 & .309821 & 0.779 & -.694065 & .5204109 \\
Cuida do Lar & -.0547798 & .3175896 & 0.863 & -.6772441 & .5676844 \\
Estudante & .0997082 & .2454848 & 0.685 & -.3814331 & .5808495 \\
Ensino Médio & $\mathbf{. 4 2 2 0 9 5 7}$ & $\mathbf{. 1 2 1 8 0 2 5}$ & $\mathbf{0 . 0 0 1}$ & $\mathbf{. 1 8 3 3 6 7 1}$ & $\mathbf{. 6 6 0 8 2 4 2}$ \\
Ensino Superior &. $\mathbf{3 4 8 9 1 3 5}$ & $\mathbf{. 1 3 8 8 7 9 7}$ & $\mathbf{0 . 0 1 2}$ & $\mathbf{. 0 7 6 7 1 4 2}$ & $\mathbf{. 6 2 1 1 1 2 7}$ \\
Casado & -.0150365 & .1234793 & 0.903 & -.2570516 & .2269785 \\
Divorciado/Separado & -.1463925 & .1687881 & 0.386 & -.4772112 & .1844261 \\
Viúvo & $\mathbf{- . 8 5 4 2 3 6 4}$ & .298122 & $\mathbf{0 . 0 0 4}$ & $\mathbf{1 . 4 3 8 . 5 4 5}$ & $\mathbf{- . 2 6 9 9 2 7 9}$ \\
Masculino & -.0122922 & .1016023 & 0.904 & -.2114291 & .1868447 \\
Idade & $\mathbf{- . 0 6 8 0 6 8 7}$ & $\mathbf{. 0 2 2 5 2 7 9}$ & $\mathbf{0 . 0 0 3}$ & $\mathbf{- . 1 1 2 2 2 2 6}$ & $\mathbf{- . 0 2 3 9 1 4 9}$ \\
Idade & $\mathbf{. 0 0 0 7 0 7 1}$ & $\mathbf{. 0 0 0 2 6 7 4}$ & $\mathbf{0 . 0 0 8}$ & $\mathbf{. 0 0 0 1 8 2 9}$ & $\mathbf{. 0 0 1 2 3 1 3}$ \\
MS & $\mathbf{- . 2 7 4 3 8 8 1}$ & $\mathbf{. 1 1 6 6 1 6 4}$ & $\mathbf{0 . 0 1 9}$ & $\mathbf{- . 5 0 2 9 5 2}$ & $\mathbf{. 0 4 5 8 2 4 3}$ \\
\hline & /cut1 & $-3,336693$ & & & \\
Pontos de Corte & /cut2 & $-2,465477$ & & & \\
\hline
\end{tabular}

Fonte: Dados da pesquisa. 
O modelo tem quatro escalas de felicidade, portanto, ao estimá-lo, obtemos três pontos de corte, que representam quando um nível de felicidade passa a ser o outro. No caso, o nível "muito infeliz" é verificado até o número -3,337, acima desse número, o indivíduo passa a ser classificado como "infeliz", até que, acima de $-2,465$, ele entra no nível "feliz", por fim, em -0,896, ele será classificado como "muito feliz". O sinal negativo não deve ser confundido com infelicidade, pois é apenas um parâmetro, ou seja, se um indivíduo com determinadas características obtiver um score -2 , isso não quer dizer que ele seja infeliz, pois o que se verifica, ao analisar os pontos de corte, é que este indivíduo estaria classificado como "feliz", de acordo com o modelo (Figura 1).

$\left|\begin{array}{|}\text { Muito Infeliz, Infeliz, Feliz Muito Feliz } \\ -3,337 \mid-2,465 \quad-0,896\end{array}\right|$

Figura 1 - Pontos de Corte do Modelo Fonte: Dados da pesquisa.

Vale destacar, como medida de verificação da qualidade do modelo, que o Wald chi ${ }^{2}$ (16) da regressão teve valor de 58,32, enquanto a Prob $>\mathrm{Ch}^{2}$ foi $<0,00001$, ou seja, com dezesseis graus de liberdade, a chance de se obter a estatística qui-quadrado $(58,32)$ é baixíssima; sendo assim, pode-se concluir que pelo menos um dos coeficientes da regressão é diferente de zero. As variáveis em negrito apresentaram significância estatística a pelo menos $5 \%$ de confiança. Na sequência, todas as variáveis do modelo são analisadas.

\subsection{Renda e felicidade}

Algumas pesquisas concluem que quanto maior a renda, maior a felicidade, mas "o resultado mais robusto e geral é que as pessoas mais ricas, na média, tendem a se considerar mais felizes, ou seja, com maior grau de bem-estar subjetivo" (CORBI; MENEZES-FILHO,
2006, p. 521). Também Lima (2007, p. 67) encontra relação positiva entre renda e felicidade, sendo que um indivíduo que possua maior renda em relação aos seus semelhantes tenderá a ter maior grau de felicidade. Scalco, Araújo e Bastos (2011) conduziram um estudo transversal na zona urbana do município de Pelotas, RS e chegaram ao resultado de que quanto maior o nível socioeconômico dos indivíduos, maior sua felicidade. Caldas (2010, p. 17) aponta que a compra de bens materiais é ineficiente para ser reproduzida em felicidade, "mesmo que bens materiais melhorem a qualidade de vida, a relação de custo/benefício é não linear, e há um ponto a partir do qual posses adicionais não melhoram a satisfação com a vida" ${ }^{2}$.

Por outro lado, segundo Borrero et al. (2013), há certo ponto em que um incremento na renda não é mais revertido em felicidade. Na realidade, o mais observado é a relação entre a pobreza acentuada e a infelicidade, mas ações de coletivismo podem amenizar esse efeito negativo.

$O$ resultado do modelo, Tabela 1 , aponta que renda não é uma variável que influencia na felicidade dos moradores de Campo Grande, MS. Além de os quartis de renda não apresentarem significância estatística, o terceiro quartil de renda tem sinal negativo, o que indicaria que as pessoas do quartil 1 seriam mais felizes. Em contrapartida, o sinal positivo dos quartis 2 e 4 mostra que um aumento na renda eleva a felicidade das pessoas. De forma geral, esses resultados apontam que não existe relação entre renda e felicidade para os moradores do município estudado.

Outra análise que pode ser feita é com relação ao efeito marginal dos quartis de renda (Tabela 2).

\footnotetext{
2 "[...] even if material goods add to quality of life, the cost/benefit relation is not linear, and there is a point past which no improvement in life satisfaction is derived from additional possessions" (CALDAS, 2010, p. 17; tradução do autor).
} 
Tabela 2 - Efeito Marginal da variável renda (em quartis)

\begin{tabular}{ccccc}
\hline Variável & Infeliz & Não muito feliz & Feliz & Muito feliz \\
\hline Quartil Renda 2 & -.0037254 & -.0135564 & -.0231798 & .0404616 \\
Quartil Renda 3 & .0001551 & .0005452 & .0008583 & -.0015586 \\
Quartil Renda 4 & -.0043572 & -.0159052 & -.0274205 & .047683 \\
\hline
\end{tabular}

Fonte: Dados da pesquisa.

A análise dos efeitos marginais também não apresenta sentido lógico para relacionar a renda com a felicidade. Se, de um lado, os que estão no segundo e quarto quartil de renda têm maior chance de serem muito felizes, os que estão no terceiro quartil têm menor probabilidade. Da mesma forma, os que estão no segundo e quarto quartil têm menos chance de serem infelizes, e os que estão no terceiro têm chance maior. Portanto, ao analisar os efeitos marginais, também não se pode afirmar que existe uma relação entre a renda e a felicidade dos entrevistados.

\subsection{Desemprego e felicidade}

Quantas vezes você já não reclamou ou ouviu alguém comentando que odeia ter que trabalhar! Isto pode parecer paradoxal, pois ao mesmo tempo em que o trabalho aparenta ser um peso para algumas pessoas "[...] pesquisas empíricas de bem-estar sugerem que o fato de perder o emprego, mesmo que seja mantido o mesmo nível de renda anterior, diminui notavelmente o nível de felicidade dos indivíduos" (CORBI; MENEZES-FILHO, 2006, p. 519). Portanto um indivíduo empregado tende a ser mais feliz que aquele que não tem emprego. Pesquisa feita por Scalco, Araújo e Bastos (2011, p. 656) apontou que "o desemprego esteve associado com menores níveis de autopercepção de felicidade". Lima (2007) também chega a essa relação entre desemprego e infelicidade.

Em nossa pesquisa, o coeficiente da variável "Empregado" foi positivo, o que mostraria que aqueles que estão empregados são, em geral, cerca de $26 \%$ mais felizes do que os que não estão, porém essa variável não apresentou significância estatística, assim, não é possível sustentar essa afirmação. A Figura 2, também analisando essa variável, mostra a relação entre o nível de felicidade e a colocação no mercado de trabalho.

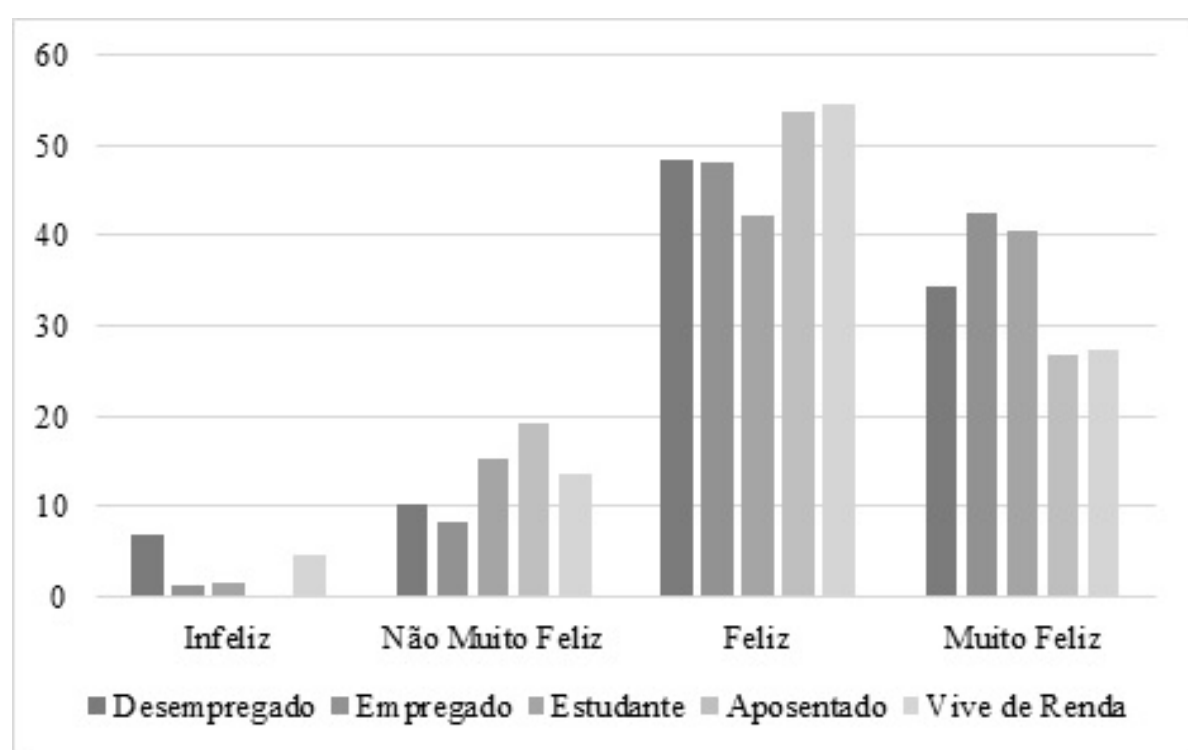

Figura 2 - Colocação no mercado e felicidade dos entrevistados Fonte: Dados da pesquisa. 
Verifica-se que os que estão empregados foram os que mais se declararam "muito felizes". Os menos "infelizes" foram os aposentados, seguidos pelos que estão empregados. Os desempregados também foram os mais "infelizes". Os efeitos marginais das variáveis relaciona- das à colocação do indivíduo no mercado de trabalho mostraram que a probabilidade de um indivíduo empregado ser muito feliz é cerca de $10 \%$ maior, o mesmo valendo para os estudantes, que apresentaram $3,87 \%$ mais chances de serem muito felizes (Tabela 3).

Tabela 3 - Efeito Marginal das variáveis relacionadas à colocação do indivíduo no mercado de trabalho

\begin{tabular}{lcccc}
\hline \multicolumn{1}{c}{ Variável } & Infeliz & Não muito feliz & Feliz & Muito feliz \\
\hline Empregado & -.0113105 & -.0369671 & -.0498953 & .0981728 \\
Aposentado & .0036096 & .0121336 & .0172234 & -.0329665 \\
Cuida do Lar & .0022078 & .007547 & .0111383 & -.0208931 \\
Estudante & -.0034871 & -.0128139 & -.0223899 & .0386909 \\
\hline
\end{tabular}

Fonte: Dados da pesquisa.

Um aposentado tem $-3,3 \%$ de chance de ser muito feliz, porém $1,7 \%$ de probabilidade de mais de ser feliz. Os indivíduos que "cuidam do lar" apresentaram cerca de $-2 \%$ de chance de serem muito felizes. Já os estudantes têm 3,9\% maior probabilidade de serem muito felizes. Embora os resultados obtidos pareçam reafirmar aqueles das pesquisas anteriores, vale ressaltar novamente que as variáveis relacionadas à colocação dos indivíduos no mercado de trabalho não apresentaram significância estatística, não se podendo concluir que tenham influência na felicidade dos entrevistados, neste trabalho.

\subsection{Estado civil e felicidade}

O estudo de Corbi e Menezes-Filho (2006, p. 534) apresentou resultados de que "indivíduos casados, de todos os países, com exceção do Japão, têm maior probabilidade de serem mais felizes que os solteiros." Com relação ao estado civil, o resultado também foi semelhante ao encontrado em pesquisas anteriores. Os solteiros seriam os mais felizes, sendo que ser casado, divorciado/separado e viúvo influenciaria negativamente na felicidade da pessoa. Porém, dessas variáveis, a única que apresentou significância estatística foi a variável "viúvo", sendo que, em média, um viúvo é cerca de $85 \%$ mais infeliz que alguém solteiro. Os casados seriam aproximadamente $1,5 \%$ menos felizes que os solteiros, e os separado/divorciados, 14,63\%. A Figura 3 mostra os percentuais dos entrevistados em relação a essas variáveis e à felicidade. 


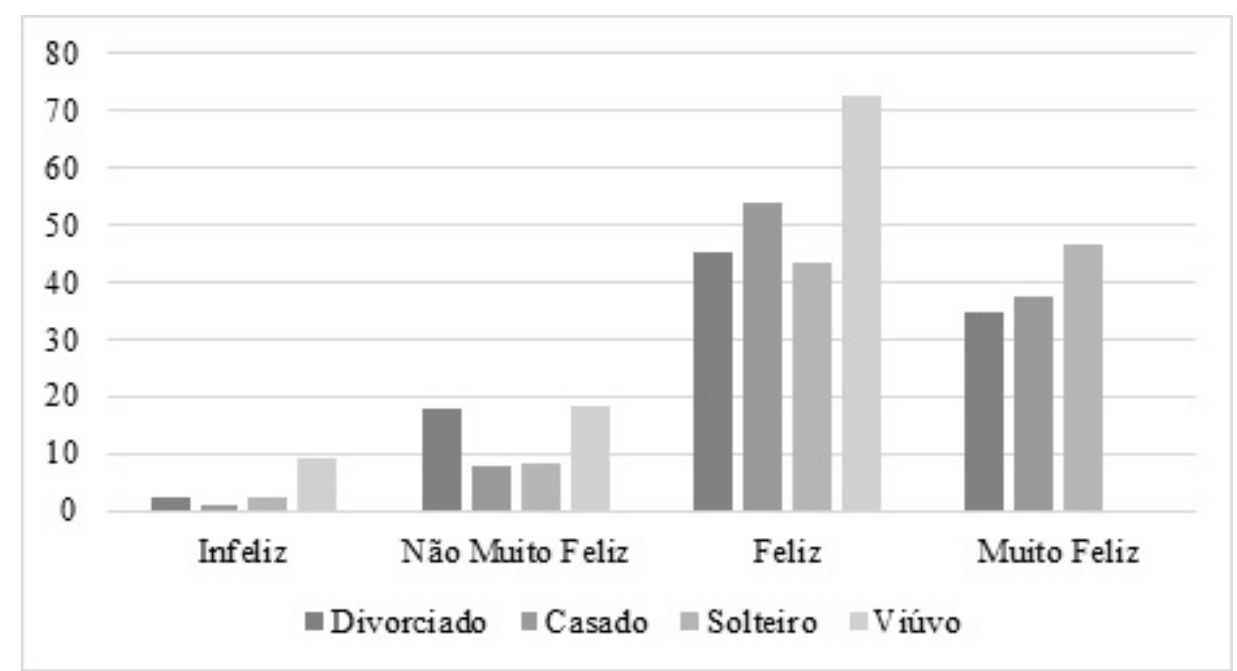

Figura 3 - Estado civil e felicidade dos entrevistados

Fonte: Dados da pesquisa.

Ao analisar a Figura 3, vemos que os solteiros são os mais "muito felizes", enquanto nenhum viúvo se declarou "muito feliz". Já os menos "infelizes" são os casados, seguidos pelos solteiros. Os efeitos marginais das variáveis mostraram que a chance de um viúvo ser muito feliz é $26,48 \%$ menor, enquanto para esse mesmo indivíduo a chance de ser não muito feliz é 15,15\% maior (Tabela 4).

Tabela 4 - Efeito Marginal das variáveis relacionadas ao estado civil dos indivíduos da pesquisa

\begin{tabular}{lcccc}
\hline \multicolumn{1}{c}{ Variável } & Infeliz & Não muito feliz & Feliz & Muito Feliz \\
\hline Casado & .0005751 & .0020206 & .0031799 & -.0057756 \\
Divorciado/Separado & .0062619 & .0207081 & .0283617 & -.0553317 \\
Viúvo & .0777718 & .1515552 & .0354383 & -.2647653 \\
\hline
\end{tabular}

Fonte: Dados da pesquisa

Um indivíduo casado tem maior probabilidade de ser feliz e cerca de $0,5 \%$ menos chance de ser muito feliz. Já os que são divorciados ou separados têm $-5,5 \%$ de chance de serem muito felizes. O que se verifica na literatura sobre o tema é que a perda de um cônjuge marca muito a vida de alguns indivíduos a ponto de não retornarem ao mesmo nível de felicidade que tinham antes do ocorrido (RODRIGUES; SILVA, 2010, p. 119).

\subsection{Localização e felicidade}

Uma variável pouco analisada em estudos anteriores foi o local de nascimento do indivíduo. O resultado esperado era que uma pessoa que morasse em um Estado diferente de sua família fosse mais infeliz em comparação com aquela que morasse no mesmo Estado. Porém o resultado do modelo indicou, com significância estatística, que aqueles que não nasceram, mas moram em Mato Grosso do Sul, são, aproximadamente, $27 \%$ mais felizes em comparação com os que nasceram e moram no Estado. 


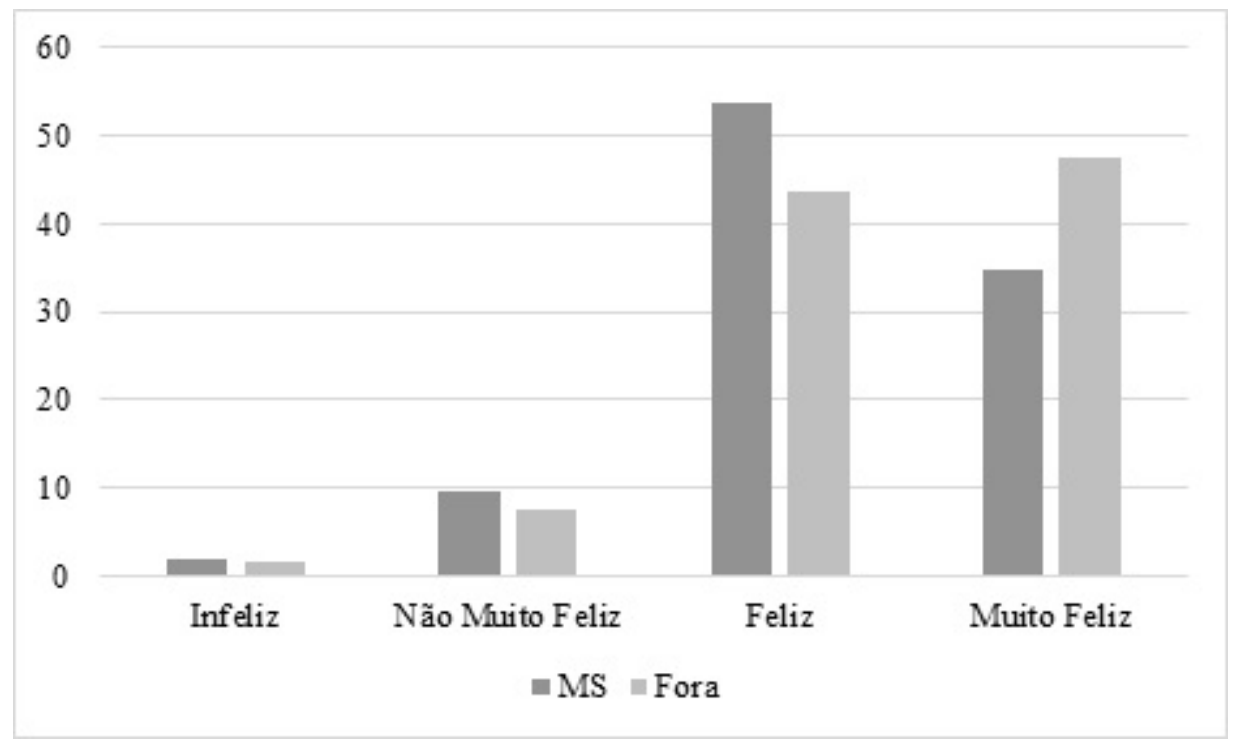

Figura 4 - Estado de origem e felicidade dos entrevistados Fonte: Dados da pesquisa.

De acordo com a Figura 4, os que moram em Mato Grosso do Sul, mas nasceram em outro Estado, são menos "infelizes" e "não muito felizes" e também são mais "muito felizes". O Efeito Marginal dessa variável também apresentou resultados que mostram que os indivíduos que nasceram e vivem no Estado de Mato Grosso do Sul têm 10,66\% menos probabilidade de serem muito felizes (Tabela 5).

Tabela 5 - Efeito Marginal da variável "nascido em MS"

\begin{tabular}{ccccc}
\hline Variável & Infeliz & Não muito feliz & Feliz & Muito Feliz \\
\hline MS & .0093705 & .0346588 & .0625432 & -.1065724 \\
\hline
\end{tabular}

Fonte: Dados da pesquisa.

Uma possível explicação para esse fenômeno está no fato de haver uma seletividade entre aqueles que migram, pois, de forma geral, para se manterem morando no Estado de destino, devem vencer certa seletividade econômica e social (BRITO; GARCIA; SOUZA, 2004, p. 6).

Portanto, pode-se supor que aquele que imigre para Mato Grosso do Sul e não consiga se estabilizar social e financeiramente acabe voltando para seu Estado de origem após certo período, e isso explicaria o coeficiente de felicidade mais elevado para os não oriundos de MS.

\subsection{Escolaridade e felicidade}

Também foi estudada a relação entre escolaridade e felicidade dos indivíduos. O modelo mostrou, com significância estatística, que aqueles que têm o ensino médio são $42,2 \%$ mais felizes que os que têm apenas o ensino fundamental; ao passo que aqueles que têm o ensino superior são $34,89 \%$ mais felizes que os que somente completaram o ensino fundamental. Esse resultado parece indicar uma relação positiva entre tempo de estudo e felicidade, mas, se olharmos com mais cautela, veremos que aqueles que têm o ensino médio são mais felizes em relação aos que só têm o ensino fundamental do que àqueles que têm o ensino superior. Ou seja, de acordo 
com o resultado, aqueles que têm o ensino superior são menos felizes do que aqueles que têm somente o ensino médio.

Os efeitos marginais das variáveis, Tabela 6, mostraram que um indivíduo que tem o ensino médio completo tem $16,38 \%$ maior probabilidade de ser muito feliz, enquanto alguém que tenha completado o ensino superior tem probabilidade $13,58 \%$ maior de ser muito feliz.

Tabela 6 - Efeito Marginal das variáveis relacionadas à escolaridade dos entrevistados

\begin{tabular}{ccccc}
\hline Variável & Infeliz & Não muito feliz & Feliz & Muito feliz \\
\hline Ensino Médio & -.0141531 & -.0523852 & -.0972756 & .1638138 \\
Ensino Superior & -.0114972 & -.0431197 & -.0811994 & .1358163 \\
\hline
\end{tabular}

Fonte: Dados da pesquisa

Embora a análise mostre que os indivíduos que não têm ao menos o Ensino Médio completo sejam menos felizes, não se pode dizer que exista uma relação linear entre anos de estudo e felicidade, sendo que a probabilidade de alguém com o Ensino Superior completo ser muito feliz é menor em comparação com um indivíduo que tenha apenas o Ensino Médio.

Além dessa evidência, Corbi e Menezes-Filho (2006) encontraram relação inversa entre escolaridade e felicidade. Helliwell (2003) obteve como resultado que a educação apresenta efeitos pequenos e insignificantes sobre o bem-estar subjetivo dos indivíduos estudados; de forma semelhante, Rodrigues e Silva (2010) também chegaram a resultado semelhante. Portanto essa é uma variável que deve ser estudada mais profundamente.

\subsection{Gênero e felicidade}

Rodrigues e Silva (2010) não encontraram relação entre gênero e felicidade nos seus entrevistados. Outros estudos apontam que as mulheres mais jovens tendem a ser mais felizes que os homens mais jovens, e as mais velhas tendem a ser mais infelizes que os homens mais velhos (MEDLEY, 1980; SPREITZER; SNYDER, 1974 apud DIENER, 1984).

De acordo com o modelo, as pessoas do gênero masculino são 1,2\% menos felizes que as do gênero feminino, mas esse resultado não apresentou significância estatística. O Efeito Marginal da variável relacionada ao gênero dos entrevistados mostrou que os indivíduos do gênero masculino têm menor chance de serem muito felizes (Tabela 7).

Tabela 7 - Efeito Marginal da variável relacionada ao gênero dos entrevistados

\begin{tabular}{ccccc}
\hline Variável & Infeliz & Não muito feliz & Feliz & Muito feliz \\
\hline Masculino & .0004704 & .0016524 & .0025983 & -.0047211 \\
\hline
\end{tabular}

Fonte: Dados da pesquisa

Em contraste, no estudo de Corbi e Menezes-Filho (2006), os indivíduos do gênero masculino apresentaram maiores chances de serem muito felizes.

\subsection{Idade e felicidade}

Na década de 1970, estudos que relacionavam idade com felicidade apon- tavam que a felicidade tendia a ser maior nos grupos mais jovens. Na sequência, com as variáveis idade, renda e saúde mais bem definidas, não foi encontrada relação significativa entre felicidade e faixa etária. No entanto estudos mais recentes, utilizando o WVS, têm sistematicamente encontrado correlação entre idade e felicidade, distribuída em formato de " $U$ ", 
sendo o pico da infelicidade em torno de 35-44 anos, e após essa faixa etária, a felicidade tende a subir até encontrar e ultrapassar os patamares encontrados nas idades mais novas (HELLIWELL, 2003, p. 342).

Neste estudo, foi encontrado resultado semelhante, as variáveis idade e idade ${ }^{2}$ apresentaram significância estatística a $1 \%$. A variável idade apresentou sinal negativo, e a variável idade ${ }^{2}$, sinal positivo, mostrando que a felicidade do indivíduo varia de acordo com sua idade. A figura 5 mostra graficamente o comportamento dessas duas variáveis, tendo como base os dados obtidos na pesquisa.

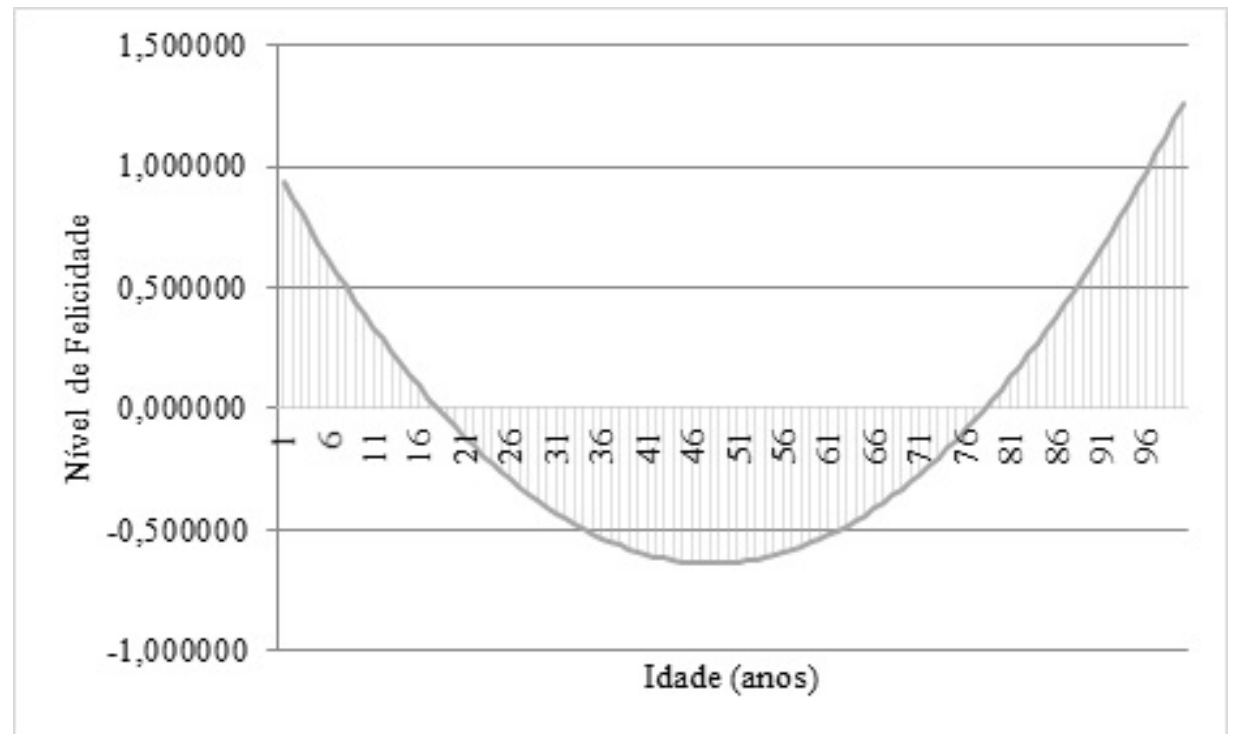

Figura 5 - Comportamento da variável idade sobre a felicidade Fonte: Dados da pesquisa.

Inicialmente, os indivíduos são mais felizes, mas, conforme vão ficando mais velhos, a felicidade vai dimuindo até que com 49 anos a felicidade (em média) volta a crescer.Corbi e Menezes-Filho (2006) também encontraram resultado semelhante e, embora os coeficientes tenham sido diferentes, ambas as variáveis apresentaram sinal igual ao obtido nesta pesquisa, portanto, ao longo do tempo, a curva de felicidade e da idade também apresentaria um formato de " $U$ ".

\section{CONCLUSÕES}

Este estudo buscou analisar a felicidade subjetiva dos morados de Campo Grande, Mato Grosso do Sul, com relação a variáveis econômicas e demográficas. Diferentemente de outros estudos, a renda não foi uma variável que explicasse a felicidade dos indivíduos, não se podendo afirmar que aqueles que têm maior ou menor renda sejam mais felizes ou infelizes.

Outro resultado importante apresentado pelo estudo, que pode ser mais bem analisado por futuros trabalhos, é a relação entre a felicidade e o Estado de origem dos indivíduos. Esse estudo mostrou que aqueles que moram em MS, mas são oriundos de outros Estados da Federação, são mais felizes que aqueles que nasceram e moram em MS. Esse é o resultado geral, mas diversos fatores podem influenciar essa análise, portanto cabe estudar o IDH, o PIB, a distância e outros variáveis relacionadas ao Estado de origem dos entrevistados.

De forma semelhante às pesquisas recentes, a idade apresentou uma curva de formato " $U$ ", sendo que a felicidade dos indivíduos tende a diminuir até por volta 
dos 49 anos de idade, passando, então, a crescer e, com cerca de 78 anos, o indivíduo atinge a mesma felicidade de um indivíduo de aproximadamente 18 anos.

A relação entre felicidade e escolaridade não apresentou relação linear, mas os mais instruídos tendem a ser mais felizes que os menos instruídos. Os que têm o ensino médio são mais felizes que aqueles que têm o ensino superior, e ambos mais do que os que têm ensino fundamental. Com relação ao estado civil, constatou-se que os indivíduos viúvos são cerca de $85 \%$ mais infelizes que os solteiros.

Esta pesquisa mostra que as variáveis que influenciam na felicidade de um indivíduo são idade, escolaridade, se o indivíduo nasceu e mora no Estado de Mato Grosso do Sul ou se é oriundo de outro Estado e mora em MS e se é ou não viúvo.

\section{REFERÊNCIAS}

BABBIE, Earl. Métodos de pesquisa survey. Tradução de Guilherme Cezarino. Belo Horizonte: UFMG, 1999. 519p. (Coleção Aprender).

BORRERO, Silvio et al .Poor and distressed, but happy: situational and cultural moderators of the relationship between wealth and happiness. Estudios Gerenciales, Cali, v. 29, n. 126, p. 2-11, já./mar. 2013.

BRITO,Fausto; GARCIA, Ricardo Alexandrino; SOUZA, Renata G. Vieira de. As tendências recentes das migrações interestaduais e o padrão migratório. ENCONTRO NACIONAL DE ESTUDOS POPULACIONAIS, 14., 20-24 set. 2004, Caxambú, MG. Anais... Caxambú, MG: Abep, 2004. Disponível em: <http:// www.abep.org.br/publicacoes/index.php/ anais/article/view/1279/1243>.

CALDAS, Silvio Borrero. The happiness-toconsumption ratio: an alternative approach in the quest for happiness. Estudios Gerenciales, Cali,v. 26, n. 116, p. 15-35, jul. 2010.

CORBI, Raphael Bottura; MENEZES-FILHO, Naércio Aquino. Os determinantes empíricos da felicidade no Brasil. Revista de Economia Política, São Paulo, v. 26, n. 4, p. 518-536, out. 2006.
DIENER, Ed. Subjective well-being. Psychological Bulletin, v. 95, n. 3, p. 542-575, jan. 1984.

FERRAZ, Renata Barboza; TAVARES, Hermano;ZILBERMAN, Monica L. Felicidade: uma revisão. Revista de Psiquiatria Clínica, São Paulo, v. 22, n. 52, p. 234-242, 2007.

GREENE, William H. Econometric analysis. 5. ed. New Jersey: Pearson Prentice Hall, 2002. 802p.

GUJARATI, Damonar N.; PORTER, DawnC. Econometria básica. 5. ed. Porto Alegre, RS: Amgh, 2011. 924p.

GUO, Teng; HU, Lingyi. Economic determinants of happiness: evidence from the US general social survey. Eprint Arxiv, p. 1-25, dez. 2011. Disponível em: <http:/ / arxiv. org/ftp/arxiv/papers/1112/1112.5802.pdf>. Acesso em: 10 jan. 2015.

HELLIWELL, John F. How'slife? Combining individual and national variables to explain subjective well-being. Economic Modeling, n. 20, p. 331-360, 2003.

HYMANN, Hebert. Planejamento e análise da pesquisa: princípios, casos e processos. 5. ed. Rio de Janeiro: Lidador, 1967. 546p.

LIMA, Sabrina Vieira. Economia e felicidade: um estudo empírico dos determinantes da felicidade no Brasil. 2007. 93f. Dissertação (Mestrado em Economia Aplicada) Universidade de São Paulo, Ribeirão Preto, SP, 2007.

NERY, Pedro Fernando. Economia da felicidade: implicações para Políticas Públicas. Brasília: Núcleo de Estudos e Pesquisas/CONLEG/ Senado, out. 2014. (Texto para Discussão n. 156).

NETTO, João Henrique Chaer Dib. A economia da felicidade: uma abordagem em pseudopainel. Informações Fipe, n. 407, p. 4753, ago. 2014.

RODRIGUES, Airton; SILVA, José Aparecido da. O papel das características sociodemográficas na felicidade. Psico-USF, v. 15, n. 1, p. 113-123, jan./abr. 2010.

SAUER, Leandro; CAMPELO, Estevan; CAPILLÉ, Maria Auxiliadora Leal. O mapeamento dos índices de inclusão e exclusão social em Campo Grande-MS: uma 
nova reflexão. Campo Grande, MS: Oeste, 2012. 68 p.

SCALCO, Diogo Luis; ARAUJO, Cora Luiza; BASTOS, João Luiz. Autopercepção de felicidade e fatores associados em adultos de uma cidade do sul do Brasil: estudo de base populacional. Psicologia: Reflexão e Crítica, v. 24, n. 4, p. 648-657, 2011. Disponível em: <http://www.scielo.br/pdf/prc/v24n4/ a04v24n4.pdf>.

\section{Sobre os autores:}

Leonardo Recena Aydos: Graduado em Ciências Econômicas e Mestre em Administração pela Universidade Federal de Mato Grosso do Sul (UFMS). E-mail: leonardoaydos@gmail.com

Leonardo Francisco Figueiredo Neto: Engenheiro Agrônomo. Mestre em Economia (USP). Doutor em Engenharia de Produção (USP). Centro de Ciências Humanas e Sociais Universidade Federal de Mato Grosso do Sul (UFMS). E-mail: lffneto@gmail.com

Wladimir Machado Teixeira: Professor Adjunto IV - UFMS. Doutor em teoria econômica pela FEA (USP). E-mail: wladimirteixeira@zipmail.com.br 